\title{
Peripheral nerve stimulation for treatment of postherpetic neuralgia: A Case report
}

\author{
Scott C. Palmer ${ }^{1}$, Alexis A. Jimenez ${ }^{2}$
}

${ }^{1}$ Department of Pain Medicine Mayo Clinic Jacksonville, Florida, USA

${ }^{2}$ Department of Pain Medicine

Mayo Clinic Rochester, Minnesota, USA

Corresponding author: Scott C. Palmer 4500 San Pablo Rd Jacksonville, FL 32224 palmer.scott@mayo.edu Tel.: + 9049563191 Fax: + 9049561750

Received: 1 April 2011 Accepted: 8 October 2011

Copyright (c) 2011 by Academy of Sciences and Arts of Bosnia and Herzegovina. E-mail for permission to publish: amabih@anubih.ba
Neuromodulation techniques have been successfully used for a variety of neuropathic pain conditions. The aim of this paper is to present a case of the successful use of a subcutaneously placed peripheral nerve stimulator for treatment of intractable postherpetic neuralgia (PHN). A 57-year old man presented with a two-year history of left thoracic pain that developed after a vesicular rash. Focal neuropathic pain had not responded to treatment with multiple analgesic medications and steroid injections. The patient had significant relief following implantation of a peripheral nerve stimulator. This case represents a contribution to the small but growing body of evidence indicating that peripheral nerve stimulation may be an effective option for treatment of PHN not responsive to less invasive modalities.

Key words: Postherpetic neuralgia, Peripheral nerve stimulation, Neuropathic pain.

\section{Introduction}

Postherpetic neuralgia is defined as pain persisting or recurring at the site of a herpes zoster rash. The annual incidence has been reported as 11 per 100,000 with a lifetime prevalence of 70 per 100,000 (1). The pain associated with PHN is notoriously difficult to treat (2).

Neuromodulation techniques have been successfully used to treat a variety of neuropathic pain conditions. Electrodes placed in the epidural space have been used to treat thoracic postherpetic neuralgia (3). Subcutaneously placed peripheral nerve stimulation leads have been used to successfully treat a wide range of conditions, including postherpetic trigeminal neuralgia (4). Published cases detailing the use of peripheral nerve stimulation(PNS) in the treatment of postherpetic radicular pain have been sparse $(5,6)$. We present a case of the successful use of PNS in the treatment of PHN. 
Neuromodulation is a reversible blockade or manipulation of pain pathways to modify physiological function and may be applied to the deep brain structures, motor cortex, spinal cord or peripheral nerves (7). The mechanism of pain relief with peripheral nerve stimulation is not clearly understood. One theory is based on the gate control theory of pain, as outlined by Melzac and Wall (8). By this theory, transmission of pain signals to the central nervous system is determined by the balance between large and small fiber activity in the dorsal horn and spinal cord, with small fiber input tending to maintain an open gate to pain transmission and large fiber input tending to close the gate.

Since electrical stimulation depolarizes large fibers before it affects small fibers, the theory suggests that it should be possible for stimulation to halt the transmission of pain signals. This explanation for the function of PNS in pain control is somewhat controversial. An alternative theory is that repetitive stimulation of peripheral nerves may produce excitation failure in c-fiber nociceptors and suppress activity in the dorsal horn of the spinal cord. Stimulation induced blockade of cell membrane depolarization may prevent axonal conduction (9). PNS may also function to decrease the release of excitatory neurotransmitters and increase the release of inhibitory neurotransmitters at the dorsal horn (10).

Spinal cord stimulation has been performed for the treatment of neuropathic pain complaints for 35 years. Though the first studies on implantable PNS therapies were produced in the 1960's, in recent years spinal cord stimulation (SCS) has been the more accepted modality for neuropathic pain (11). Early published reports of PNS showed that a minority of patients had significant pain relief.

Currently most implantable pulse generators are designated as SCS generators and are used for peripheral nerve stimulation on an "off-label" basis. Improvements in electrode and generator technology, as well as the development of percutaneous implantation techniques, have led to a resurgence of interest in the PNS modality.

Recent years have seen PNS used successfully for conditions ranging from postoperative or posttraumatic neuropathies (12) to migraine (13), low back pain (14) and fibromyalgia (15). As part of a larger study of the use of PNS for patients with facial pain, Johnson, et al. (4) reported on 4 patients treated for intractable trigeminal postherpetic neuralgia. Of the four patients, two experienced greater than $50 \%$ reduction on the visual analogue scale (VAS) score and were able to reduce their doses of analgesic medication. Harke, et al. (3) studied use of spinal cord stimulation produced via leads placed in the epidural space for treatment of postherpetic neuralgia. In this prospective trial, 23 of 28 patients experienced significant pain relief. Meglio et al. (16) retrospectively studied 10 patients who underwent trials of spinal cord stimulation for postherpetic neuralgia. Of the 10, 6 reported greater than $50 \%$ pain relief and underwent implantation. At 15 months follow-up, all 6 patients reported continued pain relief with a mean reported pain relief of $74 \%$ (16).

Review of the literature shows only three published cases of the use of PNS in the treatment of PHN related radicular pain (5, 6). Yakovlev and Peterson reported (6) a single case with improvement in pain, functional status, and medication use at six month follow up. Kouroukli et al. (5) reported on two cases with significant improvements in pain and medication use at 6 month and 3 month follow up. Our case represents the fourth reported case of the use of PNS for this indication.

\section{Case report}

A 57-year old man with a history of multiple myeloma and chronic renal insufficiency 
presented with a two year history of PHN after developing a vesicular rash in the left fifth thoracic dermatome. At the time of initial evaluation, the patient described a burning, lancinating, electrical type of pain that he rated as 9 out of 10 on the visual analogue scale (VAS). The pain was primarily focused in the left periscapular region. The patient reported a history of increased pain when air, clothing, or the shower would touch his skin. Sensory examination revealed allodynia just inferior to the left scapula. No scarring of the skin was evident. Motor function was normal. MRI of the thoracic spine showed no pathology in the region of the pain. He was treated with two series of intercostal nerve blocks, as well as an interlaminar thoracic epidural steroid injection, without relief. He had also been treated with multiple analgesic medications. A lidocaine patch was ineffective, as was a topical compounded cream of amitriptyline, gabapentin, and lidocaine. Gabapentin, pregabalin, and baclofen were tried in succession and were not tolerated due to sedation. Nortriptyline was ineffective. The use of a transcutaneous electric nerve stimulation (TENS) unit only exacerbated his pain. The patient was escalating use of oxycodone and at the time of the electrical stimulation trial was taking 60 to $80 \mathrm{mg}$ daily.

The patient agreed to undergo trial treatment with electrical stimulation therapy. During such a trial, an electrode is placed percutaneously and attached to an external pulse generator. The trial implantation is performed under minimal conscious sedation in order to determine the location of the paresthesia produced and to determine the patient's response to simulation. The patient is then typically discharged with the epidural lead in place with a follow-up visit scheduled several days later. At the time of follow up, the patient's response to stimulation is recorded and the percutaneous lead is removed. Significant pain relief during such a trial indicates that the patient will likely have a good outcome from permanent stimulator implantation. In this case, the trial was carried out with placement of both epidural and subcutaneous leads.

After carefully outlining the area of pain and obtaining informed consent, the patient was taken to the surgical suite. He was placed in a prone position and the lower and mid back was prepped and draped in a sterile fashion. The patient was under light conscious sedation and was monitored throughout the case. After anesthetizing the skin, a 14 gauge Tuohy needle was placed into the epidural space under fluoroscopic guidance at the T12-L1 level using loss of resistance technique. An eight contact electrode (Octrode $^{\mathrm{TM}}$ ) lead (St Jude Medical, Plano, TX) was placed through the Tuohy needle, into the epidural space, with the most superior lead at the midportion of the T1 vertebral body, $0.5 \mathrm{~mm}$ to the left of midline. The lead was attached to a St. Jude pulse generator and paresthesia was produced covering the area of left thoracic pain. A second identical lead was placed percutaneously through a 14 gauge Tuohy needle into the subcutaneous space in the area of the left T5 dermatome. The electrode was placed medial to and slightly superior to the location of his most significant pain and allodynia. This electrode was attached to the pulse generator and was tested with the epidural lead inactivated. The subcutaneous lead also produced paresthesia in the distribution of his pain. Tuohy needles and styletes were removed from each site and the percutaneous leads were sutured to the skin. The patient was seen in the recovery room for further fine tuning of programming. The epidural and subcutaneous lead both proved to be equally effective, producing paresthesia and relief of the patient's thoracic pain. The patient was discharged for a 72 hour trial period. He was provided with programs to activate both electrodes individually or both 
simultaneously. During the trial period, the patient reported $75 \%$ reduction in pain and his opioid requirements only amounted to $25 \%$ of his typical daily use. The patient reported equivalent pain relief regardless of which electrode was activated. At the time of follow up, both percutaneous leads were removed.

Six weeks after the trial period, the patient returned for permanent implantation. He had a history of coagulopathy, likely due to his history of multiple myeloma and treatment with chemotherapeutic agents. Due to bleeding during the procedure, the decision was made to proceed with implantation of subcutaneous leads, rather than to perform a laminectomy for epidural placement. The implantation was performed under general anesthesia with assistance from a neurosurgeon. The initial percutaneous lead was placed in a location identical to the subcutaneous trial lead. In order to provide additional coverage of any more lateral symptoms, a second subcutaneous lead was placed approximately 3 inches lateral to the first lead, in the path of the T5 dermatome. A subcutaneous pocket was produced in the upper left buttock and a St Jude Eon Mini rechargeable implantable pulse generator was implanted.

At 6 months follow-up, the patient reported decreased pain and improved quality of life. The maximum pain level had diminished from $10 / 10$ to $4 / 10$ on VAS. The constant burning pain had been completely alleviated but he continued to report occasional lancinating pain. At the time of the last follow-up, he had decreased his use of oxycodone 5 to $10 \mathrm{mg}$ a day.

\section{Discussion}

We report a case of the successful use of a subcutaneously placed peripheral nerve stimulator for the treatment of postherpetic neuralgia. This adds to a very small but growing body of literature indicating that peripheral nerve stimulation may be a useful option in the treatment of this relatively common and potentially disabling condition. This study is, of course, limited in that it is a single case report. Prospective studies are needed to establish the utility and safety of PNS for the management of postherpetic neuralgia. The use of PNS entails the risk of nerve injury, bleeding or infection, as well as lead migration or fracture (17). PNS treatment carries a significant up front cost. A cost benefit analysis of SCS and PNS for treatment of chronic pain showed that cost benefits began to accrue after two years of stimulator use (18). There is insufficient data to make a similar claim for the use of PNS alone for chronic pain or for either modality for this particular indication. One clinical limitation of our case was the placement of an additional subcutaneous lead during the permanent implantation. The decision to place this additional lead was made during the surgical procedure when bleeding led to aborting the placement of the epidural lead. Placement of a stimulator lead without trial on a conscious patient may entail additional procedural cost with uncertain clinical efficacy. At this stage in the use of PNS for the treatment of neuropathic pain, guidelines for the correct position or number of subcutaneous leads required or the proper duration of the trial period have not been clearly established.

\section{Conclusion}

PNS may be an effective treatment option for patients who have not responded to more conservative treatment. Prospective studies may be useful to determine the long term efficacy of this technology in the treatment of post-herpetic neuralgia, as well as to determine cost effectiveness.

Conflict of interest: The authors declare that they have no conflict of interest. This study was not sponsored by any external organization. 
Authors' contributions: Conception and design: SCP; Acquisition, analysis and interpretation of data: SCP; Drafting the article: SCP, AAJ; Revising it critically for important intellectual content: SCP, AAJ.

\section{References}

1. MacDonald BK, Cockerell OC, Sander JWAS, Shoryon SD. The incidence and lifetime prevalence of neurological disorders in a prospective community-based study in the UK. Brain. 2000;123:665-76.

2. Benzon HT, Kiran C, Darnule A, Chung B, Wille $\mathrm{O}$, Malik K. The prevention and management of postherpetic neuralgia with emphasis on interventional procedures. Regional Anesthesia and Pain Medicine. 2009;34(5)514-21.

3. Harke H, Gretenkort P, Ladleif H,. Koester P, Rahman S. Spinal cord stimulation in postherpetic neuralgia and in acute herpes zoster pain. Pain Med. 2002;94:694-700.

4. Johnson M, Burchiel K. Peripheral stimulation for treatment of trigeminal postherpetic neuralgia and trigeminal posttraumatic neuropathic pain: a pilot study. Neurosurgery. 2004;55:135-42.

5. Kouroukli I, Neofytos D, Panaretou V,Zompolas V, Papastergiou D, Sanidas G, et al. Peripheral subcutaneous stimulation for the treatment of intractable postherpetic neuralgia: two case reports and literature review. Pain Pract. 2009;9:225-9.

6. Yakovlev A, Peterson A. Peripheral nerve stimulation in treatment of intractable postherpetic neuralgia. Neuromodulation. 2007;10:373-5.

7. Bittar RG, Teddy PJ. Peripheral neuromodulation for pain. J Clin Neurosci. 2009;16:1259-61.

8. Melzack R, Wall PD. Pain Mechanisms: A new theory. Science. 1965;150:971-9.
9. Dorsi M J, Belzberg A J. Peripheral nerve stimulation. In: Fishman S, Ballantyne J, Rathmell J, editors. Bonica's Management of Pain, 4th edition, Philadelphia: Wolters Kluwer/Lippincott Williams and Wilkins; 2010. p. 1375-8.

10. Cui JG, O'Connor WT, Ungerstedt U, Linderoth B, Meyerson BA. Spinal cord stimulation attenuates augmented dorsal horn release of excitatory amino acids in mononeuropathy via a GABAergic mechanism. Pain. 1997;73(1):87-95.

11. Slavin K. Peripheral nerve stimulation for neuropathic pain. Neurother. 2008;5:100-6.

12. Mobbs RJ, Nair S, Blum P. Peripheral nerve stimulation for the treatment of chronic pain. J Clin Neurosci. 2007;14:216-221.

13. Weiner RL. Occipital neurostimulation for treatment of intractable headache syndromes. Acta Neurochir -Suppl (Wien). 2007;97:129-33.

14. Paicius RM, Bernstein CA, Lempert-Cohen C. Peripheral nerve field stimulation for the treatment of chronic low back pain: preliminary results of long-term follow up: a case series. Neuromodulation. 2007;10:279-90.

15. Thimineur M, De Ridder D. C2 area neurostimulation: A surgical treatment for fibromyalgia. Pain Med. 2007;8:639-46.

16. Meglio M, Cioni B, Prezioso A, Talamonti G. Spinal cord stimulation (SCS) in the treatment of postherpetic pain. Acta Neurochir Suppl (Wien). 1989;46:65-6.

17. Deer TR, Levy RM, Rosenfeld EL Prospective clinical study of a new implantable peripheral nerve stimulation device to treat chronic pain. Clin J Pain. 2010;26(5):359-72.

18. Nagy AM, Aeschbach A, Stanton-Hicks M. Cost benefit analysis of neurostimulation for chronic pain. Clin J Pain. 2004;20(6):462-8. 